\title{
Exposure to Sodium Salicylate Disrupts VGLUT3 Expression in Cochlear Inner Hair Cells and Contributes to Tinnitus
}

\author{
Wei ZHANG ${ }^{1,2}$, Zhe PENG ${ }^{1}$, Shukui YU ${ }^{1}$, Qing-Ling SONG ${ }^{1}$, Teng-Fei QU ${ }^{1}$, Ke LIU ${ }^{1}$, \\ Shu-Sheng GONG ${ }^{1}$
}

${ }^{1}$ Department of Otolaryngology - Head and Neck Surgery, Beijing Friendship Hospital, Capital Medical University, Beijing, China, ${ }^{2}$ Department of Otolaryngology - Head and Neck Surgery, Eye Hospital China Academy of Chinese Medical Science, Beijing, China

Received April 8, 2019

Accepted August 15, 2019

Epub Ahead of Print December 19, 2019

\section{Summary}

To examine whether exposure to sodium salicylate disrupts expression of vesicular glutamate transporter 3 (VGLUT3) and whether the alteration in expression corresponds to increased risk for tinnitus. Rats were treated with saline (control) or sodium salicylate (treated) Rats were examined for tinnitus by monitoring gap-pre-pulse inhibition of the acoustic startle reflex (GPIAS). Auditory brainstem response (ABR) was applied to evaluate hearing function after treatment. Rats were sacrificed after injection to obtain the cochlea, cochlear nucleus (CN), and inferior colliculus (IC) for examination of VGLUT3 expression. No significant differences in hearing thresholds between groups were identified $(p>0.05)$. Tinnitus in sodium salicylate-treated rats was confirmed by GPIAS. VGLUT3 encoded by solute carrier family 17 members 8 (SLC17a8) expression was significantly increased in inner hair cells (IHCs) of the cochlea in treated animals, compared with controls $(p<0.01)$. No significant differences in VGLUT3 expression between groups were found for the cochlear nucleus $(\mathrm{CN})$ or IC $(p>0.05)$. Exposure to sodium salicylate may disrupt SLC17a8 expression in IHCS, leading to alterations that correspond to tinnitus in rats. However, the $\mathrm{CN}$ and IC are unaffected by exposure to sodium salicylate, suggesting that enhancement of VGLUT3 expression in IHCs may contribute to the pathogenesis of tinnitus.

\section{Key words}

Vesicular glutamate transporter 3 - Peripheral auditory system • Auditory cortex $\bullet$ Tinnitus $\bullet$ Sodium salicylate

\section{Corresponding authors}

Z. Peng (feidaxibao@sina.com) or K. Liu (Keliu66@hotmail.com) or S.-S. Gong (gongss1962@163.com), Department of Otolaryngology - Head and Neck Surgery, Beijing Friendship Hospital, Capital Medical University, Beijing City Xi Cheng District Road 95 Yong An, Beijing, 100050, China

\section{Introduction}

Tinnitus is a subjective perception of sound in the ear in the absence of external stimulation. It has been proposed that about $10-20 \%$ of the general population suffer from tinnitus (Baguley et al. 2013). A previous study showed that tinnitus may significantly reduce quality of life for millions of people, sometimes even leading to suicide in individuals with severe tinnitus (Shargorodsky et al. 2010). Tinnitus is therefore considered a serious type of auditory disorder worldwide. Unfortunately, no effective therapies are available for individuals suffering from tinnitus, as the underlying mechanism remains unclear. Tinnitus may be induced by an imbalance between the release of excitatory vs. inhibitory neurotransmitters in the peripheral and central auditory systems. Glutamate, the most critical excitatory neurotransmitter in the auditory system, is confirmed to be responsible for delivery of sound-encoding information from the cochlea to the brain ( $\mathrm{Lu} 2014)$.

Exposure to sodium salicylate (SS) was demonstrated previously to cause tinnitus (Chen et al. 2013). SS, the active component of the non-steroidal 
anti-inflammatory drug aspirin is commonly used to manage rheumatoid arthritis. Therapeutic doses of aspirin inhibit cyclooxygenase and decrease the synthesis of prostaglandin. The main metabolites of salicylate, the active ingredient of SS, are salicylic acid and glucuronic acid. Tinnitus induced by high doses of SS was previously characterized as temporary hearing loss and shown to be reversible (Sun et al. 2015). Tinnitus induced by SS was reported to be related to damage to cochlear hair cells and spiral ganglion cells (Norena 2015). Additional findings associated with SS-induced tinnitus include changes in the balance between excitatory and inhibitory neurotransmitter release and receptor modification (Richardson et al. 2012). For example, tinnitus has been associated with the downregulation of decreased Gamma-aminobutyric acid (GABA) receptor expression in the inferior colliculus was found to be associated with tinnitus (Bauer 2000). Alternatively, exposure to SS may cause extensive accumulation of arachidonic acid and N-methyl-Daspartic acid (NMDA) receptor activation, resulting in excessive excitability (Ruel et al. 2008).

VGLUT3, which is encoded by SLC17a8, has been reported to have tight connection with hearing (Seal 2008), suggesting that VGLUT3 may also be a critical factor involved in the process of tinnitus. VGLUT3 is known to play a role in hearing loss. VGLUT3 is specifically expressed in IHCs of the peripheral auditory system and is widely expressed in the brain (coupled with GABA transporters) (Stensrud et al. 2013). Variations in the expression of VGLUT3 in cochlear IHCs and outer hair cells (OHCs) may be due to their varying functions and susceptibility to trauma (Amarjargal et al. 2009). Tinnitus is considered to be a phantom restricted to the auditory cortex. Emerging evidence suggests that changes in levels of neurotransmitters in the peripheral auditory system may be involved in the generation of tinnitus (Norena 2013).

We postulated that VGLUT3 expressed in the peripheral auditory system may contribute to tinnitus induced by exposure to SS. In this study, various approaches, including a tinnitus animal model, behavior testing, hearing detection, and immunochemistry, were applied to examine the role of exposure to SS in tinnitus. We found that exposure to SS can induce tinnitus and specifically disrupted expression of VGLUT3 in the cochlear IHCs, but not the cochlear nucleus or inferior colliculus. These results suggest that the changes of
VGLUT3 expression in the peripheral auditory system contribute to the SS induced tinnitus.

\section{Materials and Methods}

\section{Animal model}

All studies were approved by the Institutional Animal Care and Use Committee at the Capital Medical University of China. Adult male Wistar rats weighing 250-300 g were obtained from the animal experimental ministry of Capital Medical University. Rats were divided in two groups based on injection contents. Animals in the control group received intraperitoneal injections of saline once daily for ten days $(n=12)$. Rats in the treatment groups received intraperitoneal injections of $5 \%(200 \mathrm{mg} / \mathrm{kg}) \mathrm{SS}$ once daily for ten days. All rats were housed in standard home cages for one week prior to receiving peritoneal injections $(n=12)$.

\section{Behavioral testing}

Animals were placed in a permeable sound box resting on a sensitive piezoelectric transducer capable of generating a voltage proportional to the magnitude of the startle reflex. The startle reflex was evoked by sound stimuli generated digitally by a digital signal processor. The test apparatus was located in a soundproof chamber equipped with a tweeter on the chamber's ceiling, approximately $10 \mathrm{~cm}$ above the rat's head (Coulbourn Instruments, USA). Animals were placed in the box for $10 \mathrm{~min}$ prior to testing for adaptation. Test sessions included ten gap and ten no-gap trials pair-arranged with pure tone background (70 dB SPL, $12 \mathrm{kHz}$ and $16 \mathrm{kHz}$, 12-21 s, randomly). The gap lasting $75 \mathrm{~ms}$ was embedded in the tone $100 \mathrm{~ms}$ prior to the startle stimulus (120 dB SPL, broad-banded noise, $20 \mathrm{~ms}$ ) (Fig. 1A). The maximum startle reflex within $250 \mathrm{~ms}$ after the startle pulse was recorded. Gap pre-pulse inhibition of acoustic startle was calculated as a ratio: 1 - (gap/no-gap).

Rats treated with SS that showed signs of tinnitus had poorer gap detection ability. This decrease in gap detection can be measured as a lack of suppression of the startle reflex during trials that contain a brief silent gap before the startle stimulus (Edward 2013). Tinnituspositive rats will have a lower gap pre-pulse inhibition ratio. The behavior test was carried out $2 \mathrm{~h}$ after SS treatment. We compared the inhibition ratio of SS-treated animals to that of animals in the control group. 
A

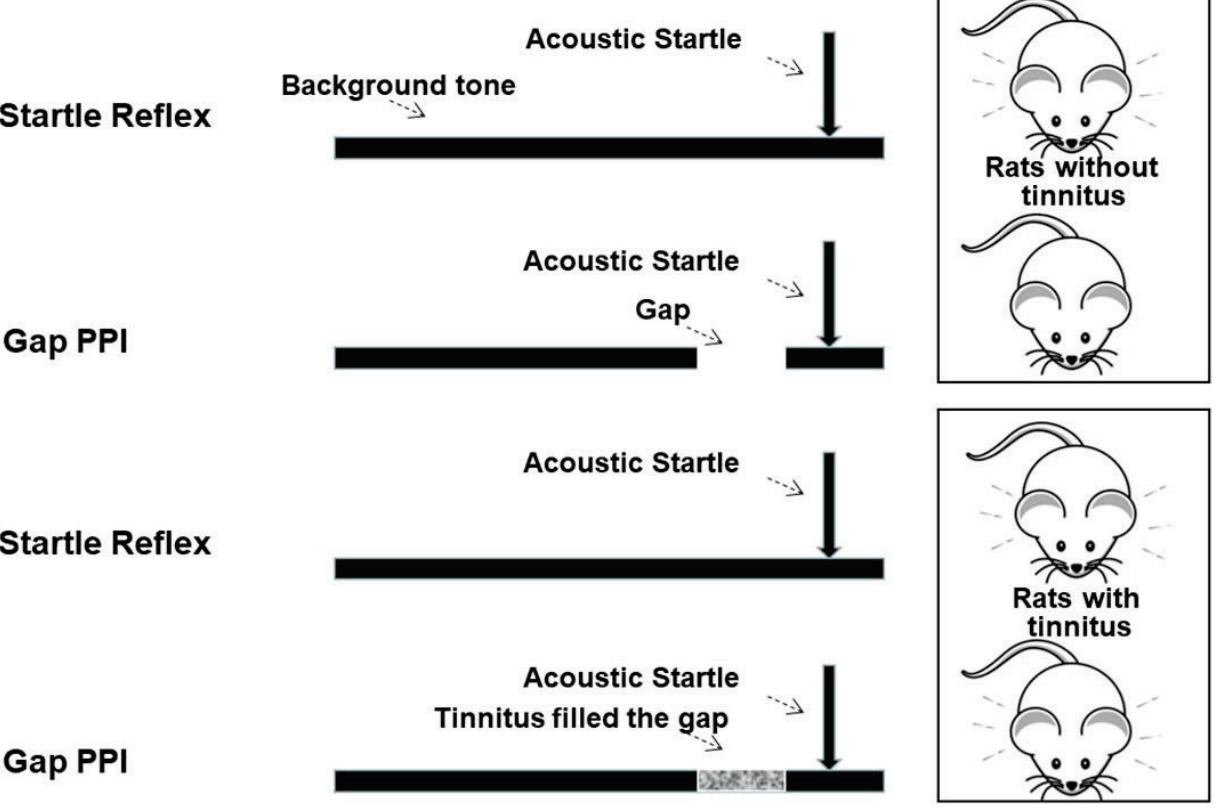

B

\begin{tabular}{clccc}
\hline Groups & \multicolumn{2}{c}{$\begin{array}{c}\text { Gap PPI mean inhibition rate } \\
(\text { ratio, } \mathrm{n}=12)\end{array}$} & $\begin{array}{c}\text { Amplitude of startle reflex } \\
(\mathrm{mV}, \mathrm{n}=12)\end{array}$ \\
\cline { 2 - 4 } & Mean $\pm \mathrm{SD}$ & $p$ value & Mean $\pm \mathrm{SD}$ & $p$ value \\
\hline Control group & $0.68 \pm 0.047$ & 0.02 & $\frac{170.08 \pm 28.61}{274.91 \pm 53.36}$ & 0.001 \\
\hline Treated group & $0.51 \pm 0.045$ & 0.02 & & \\
\hline
\end{tabular}

Fig. 1. Graphical schema and results of tinnitus behavioral testing. (A) Graphical schema of tinnitus screening by GPIAS. Background pure tones were $12 \mathrm{kHz}$ and $16 \mathrm{kHz}$ at intensity of $70 \mathrm{~dB}$ SPL. The inter-trial interval time was $12-21 \mathrm{~s}$, randomly. The acoustic startle stimulus was broad-banded noise of 20 ms at intensity of $120 \mathrm{~dB}$ SPL. (A) The gap lasting $75 \mathrm{~ms}$ was set at 100 ms ahead of acoustic startle stimulus. Animals were startled when there was an acoustic startle stimulus, as shown in the first line. After several trials, the animals without tinnitus presented the startle inhibition, as shown in the second line. However, animals with tinnitus had a deficit in the startle inhibition, as shown in the second line and presented a decrease in inhibition rate. (B) Mean inhibition rate of gap PPI and amplitude of the startle reflex. There were significant differences in the GPIAS and amplitude of the startle reflex between the control and treated groups.

\section{Auditory brainstem response recordings}

The evoked response signal-processing (TDT) system was used to measure the ABR. Animals were anesthetized with choral hydrate $(6 \%, 0.6 \mathrm{ml} / 100 \mathrm{~g})$ and placed in a soundproof chamber. Three needle electrodes were inserted subcutaneously at the vertex (active) and beneath each pinna (reference and ground). Closed-field acoustic stimulation was used, and hearing status was evaluated at the end of the study. The ABR thresholds at pure tone frequencies of $2,4,8,12,16,20$, and $24 \mathrm{kHz}$ (tone bursts of $5 \mathrm{~ms}$ duration, $0.5 \mathrm{~ms}$ cosine ramps, $21 \mathrm{~Hz}$ repetition rate) were recorded.

\section{Immunofluorescence}

Animals were decapitated after anesthesia with choral hydrate. Temporal bones were removed and fixed for $2 \mathrm{~h}$ in $10 \%$ formaldehyde in phosphate-buffered saline (PBS). The central auditory system (cochlear nucleus and inferior colliculus) were quickly removed and kept at $-80^{\circ} \mathrm{C}$. Auditory sensory epithelia were dissected, then treated with $0.3 \%$ Triton X-100 (Sigma, St. Louis, MO, USA) in PBS for $30 \mathrm{~min}$. Non-specific binding was blocked with $10 \%$ normal goat serum (Jackson ImmunoResearch, West Grove, PA, USA) for $1 \mathrm{~h}$ at room temperature. Tissues were incubated overnight at $4{ }^{\circ} \mathrm{C}$ with the following primary antibodies: rabbit anti-myosin VIIa (1:300, Proteus Biosciences Inc., Ramona, CA, USA) and guinea pig anti-VGLUT3 (1:300, Millipore, Burlington, MA, USA). After rinsing in PBS, the samples were incubated in fluorescent labeled secondary antibodies (Alexa Fluor 488 and 647, Invitrogen/Molecular Probes, Carlsbad, CA, USA) for $1 \mathrm{~h}$ at room temperature. Nuclei were visualized with 4',6-diamidino-2-phenylindole (DAPI) (AppliChem, Darmstadt, Germany). 


\section{Confocal microscopy}

Laser scanning confocal microscopy was conducted with a $\times 63$ oil immersion objective lens (LEICA TCS SP8) using the excitation wavelengths of 488 and $647 \mathrm{~nm}$. Local images were digitally magnified two-fold. Sequence scanning was performed from the apex from each ear, at an interval of $0.3 \mu \mathrm{m}$. All digital images were captured under identical exposure and light settings. Images were processed into a virtual file format, then transferred to the Definiens share. A Definiens zoom cache was created, and the virtual slides were then uploaded, and rule sets were created using the Definiens software.

\section{Western blot}

Total protein was extracted from samples taken from six rats. Protein concentration was determined using an ultraviolet spectrophotometer (Thermo, MultiSkan3). Samples were used to electrophorese the VGLUT3 protein. Equal quantities of protein $(20 \mu \mathrm{g})$ were loaded into the gel and separated by electrophoresis. Next, the proteins were transferred onto nitrocellulose membranes (Millipore, Billerica, MA, USA) and blocked with 3\% bovine serum albumin in Tris-buffered saline, $0.1 \%$ Tween 20 (TBS-T; CWbio. Co. Ltd., Beijing, China). The membranes were incubated with the primary antibody overnight and washed in TBS-T. Secondary antibodies were diluted in TBST and incubated with membranes for $2 \mathrm{~h}$ at room temperature. Finally, the immunoreactive bands were visualized with electrochemiluminescence (ECL; Millipore, Billerica, MA, USA). Western blot images were quantified using the Image Lab software. Band intensities of VGLUT3 were expressed relative to GAPDH. The following antibodies were used: 1:1,000 mouse polyclonal antiVGLUT3 (s34-34, Abcam, Cambridge, UK) and 1:5,000 goat anti-mouse Ig GHRP (Jackson, Ely, UK).

\section{Statistical analysis}

All data are presented as mean \pm SD. Statistical analysis of the optical density (OD) values was conducted using the independent $t$-test. ABR threshold shifts were analyzed using two-way analysis of variance (ANOVA), followed by Bonferroni's multiple-comparisons test. $p<0.05$ were considered statistically significant.

\section{Results}

\section{Behavioral assessment}

To generate an animal model of tinnitus, intraperitoneal injections of SS $(200 \mathrm{mg} / \mathrm{kg})$ were administered once daily for 10 days. We performed a gap pre-pulse inhibition test of the acoustic startle reflex (GPIAS) in the treated and control groups. Mean inhibitory rates differed significantly between the treated and control groups $(0.51 \pm 0.045$ vs. $0.68 \pm 0.047$, respectively; $p<0.05$ ), as shown in Fig. 1B. Next, the acoustic startle reflex was assessed in SS-treated and untreated (control) groups. Mean amplitudes of the startle reflex in the treated and control animals were $274.91 \pm 53.36$ and $170.08 \pm 28.61 \mathrm{mV}$, respectively (Fig. 1B). Amplitude was significantly higher in the treated group than the control group $(p=0.001)$. These observations are consistent with those previously reported in the literature (Chen et al. 2013). These results indicate that exposure to SS $(200 \mathrm{mg} / \mathrm{kg})$ once daily for 10 days can induce tinnitus in rats, and hyperacusis is often accompanied by tinnitus.

\section{Hearing thresholds}

To determine whether exposure to SS at a dose of $200 \mathrm{mg} / \mathrm{kg}$, once daily for 10 days induced significant hearing loss, we measured ABR thresholds in both treated and control groups. ABR testing at pure tone frequencies of 2, 4, 8, 12, 16, 20, and $24 \mathrm{kHz}$ was carried out $2 \mathrm{~h}$ after the last injection on the $10^{\text {th }}$ day. The ABR threshold was defined as the lowest stimulus intensity that produced reliable and reproducible (in at least two trials) ABR waves. Thresholds in the treated group were $35.62 \pm 4.95, \quad 33.12 \pm 5.93, \quad 35.00 \pm 5.34, \quad 33.12 \pm 5.93$, $31.25 \pm 3.53, \quad 35.00 \pm 5.34$, and $47.5 \pm 11.64 \mathrm{~dB}$ sound pressure level (SPL). Thresholds in the control group were $31.42 \pm 3.77,30.00 \pm 2.88,25.71 \pm 1.88,25.00 \pm 4.08$, $26.42 \pm 5.56,30.00 \pm 4.08$, and $33.57 \pm 5.56 \mathrm{~dB}$ SPL. There were no significant differences between the treated and control groups at 4,16 , or $20 \mathrm{kHz}$ (Fig. 2, $p>0.05$ ). However, significant differences were found at 8,12 , and $24 \mathrm{kHz}$ (Fig. 2, $p<0.05$ ). Maximum elevations of the ABR threshold were observed at 8, $12(<10 \mathrm{~dB}$ SPL $)$, and $24 \mathrm{kHz}$ (nearly $10 \mathrm{~dB}$ SPL) (Fig. 2), suggesting that exposure to SS at doses used in this study did not affect the results of behavioral testing.

\section{Exposure to SS increased VGLUT3 expression in IHCs}

To examine whether exposure to SS affected VGLUT3 expression in the hearing pathway, we examined VGLUT3 expression in cochlear hair cells, cochlear nucleus, and inferior colliculus. As shown in Fig. 3A, there was no positive immunostaining in the $\mathrm{OHCs}(\mathrm{OHC1}-3)$, 


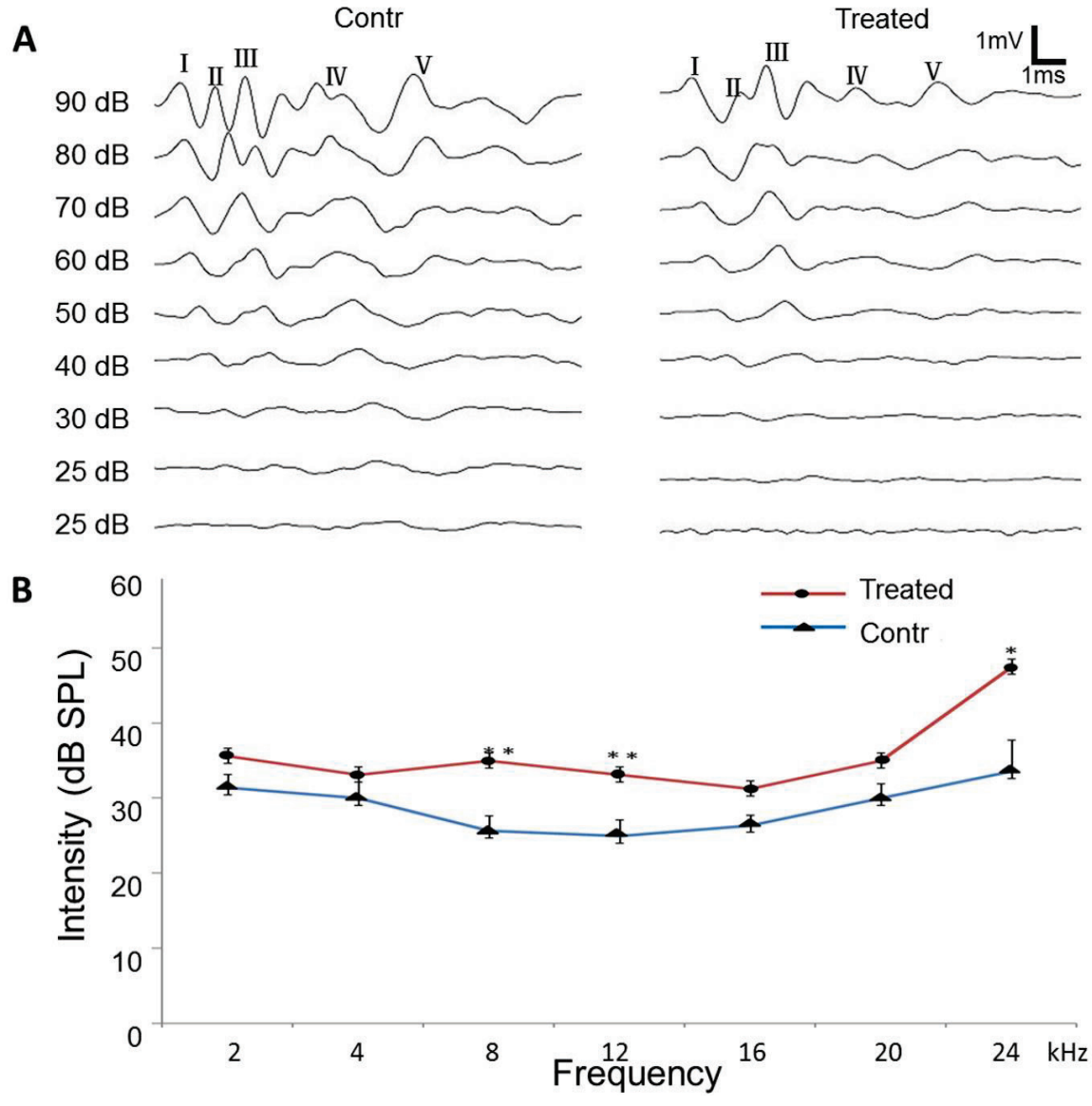

Fig. 2. Examinations of hearing threshold after exposure to salicylate sodium. (A) Representative waveforms of the auditory brainstem response (ABR) evoked by pure tones at frequencies of $2,4,8,12,16,20$, and $24 \mathrm{kHz}$ in the control (upper left) and salicylate-treated rats (upper right). Wave III was used to measure ABR thresholds. (B) Mean ABR thresholds at 2, 4, 8, 12, 16, 20, and $24 \mathrm{kHz}$ were detected in the control and treated groups. Blue line indicates the control group; red line indicates the treated group. There were no significant differences between the control and treated groups at 2, 4, 16, or $20 \mathrm{kHz} \quad(p>0.05)$. Significant differences were found at 8,12 , and $24 \mathrm{kHz}$. Maximal elevation of the ABR threshold was nearly $10 \mathrm{~dB}$ SPL at 8, 12 , and $24 \mathrm{kHz}$ (control group, $\mathrm{n}=7$; treated group, $\mathrm{n}=8) ;{ }^{*} p<0.05$, ${ }^{* *} p<0.01$.

suggesting that VGLUT3 was not expressed in OHCs. These observations were consistent with results reported previously indicating that VGLUT3 was absent in OHCs. In this study, we found specific and significant expression of VGLUT3 in cochlear IHCs (Fig. 3A-B), as previously reported (Seal 2008). The OD values were 12.21 \pm 1.59 and $8.71 \pm 2.19$, respectively (Fig. 3C). Significant upregulation of VGLUT3 was observed in the IHCs of animals in the treated group, as compared with controls (Fig. 3C). To confirm the upregulation of cochlear VGLUT3 expression, Western blot analysis was performed (Fig. 4A). The OD values (VGLUT3/GAPDH) were $0.4553 \pm 0.0061$ and $0.7130 \pm 0.0168$, respectively, (Fig. 4B), suggesting that SS treatment increased VGLUT3 levels in IHCs, which contributed to the development of tinnitus.

Exposure to SS did not disrupt VGLUT3 expression in cochlear nucleus and inferior colliculus

Next, we examined whether SS treatment affected VGLUT3 expression in the cochlear nucleus and inferior colliculus. Figure 5 shows positive VGLUT3 expression in the neuronal cells of the cochlear nucleus. The OD values of VGLUT3 expression in the treated and control groups were estimated to be $35.96 \pm 1.34$ and $38.15 \pm 2.17$, respectively. There were no significant differences in VGLUT3 expression between treated and control groups (Fig. 5A, B, $p>0.05$ ). Western blot analysis was performed to further confirm VGLUT3 expression in the cochlear nucleus. The OD values obtained (VGLUT3/GAPDH) were $0.03217 \pm 0.004651$ and $0.02550 \pm 0.003958$, respectively, indicating no significant difference between the treated and control animals (Fig. 5C, D, $p>0.05$ ), as noted in the staining data shown in Fig. $5 \mathrm{~A}$ and $\mathrm{B}$.

Next, we measured VGLUT3 expression in the inferior colliculus, a critical structure of the auditory center. Figure 6 shows positive VGLUT3 expression in the neurons of the inferior colliculus in the treated and control groups. The OD values of VGLUT3 expression in the treated and control groups were $1.747 \pm 0.03$ and $1.630 \pm 0.05$, respectively; there were no significant differences between the treated and control animals (Fig. 6A-B, $p>0.05$ ). The results of Western blot were consistent with those of immunostaining (Fig. 6C-D). The OD values (VGLUT3/GAPDH) were $0.03074 \pm$ 0.005713 and $0.02531 \pm 0.003904$, respectively, with no significant difference between treated and control animals. The results presented above suggest that VGLUT3 expression in the cochlear nucleus and inferior colliculus remained unaffected by SS treatment. 
A
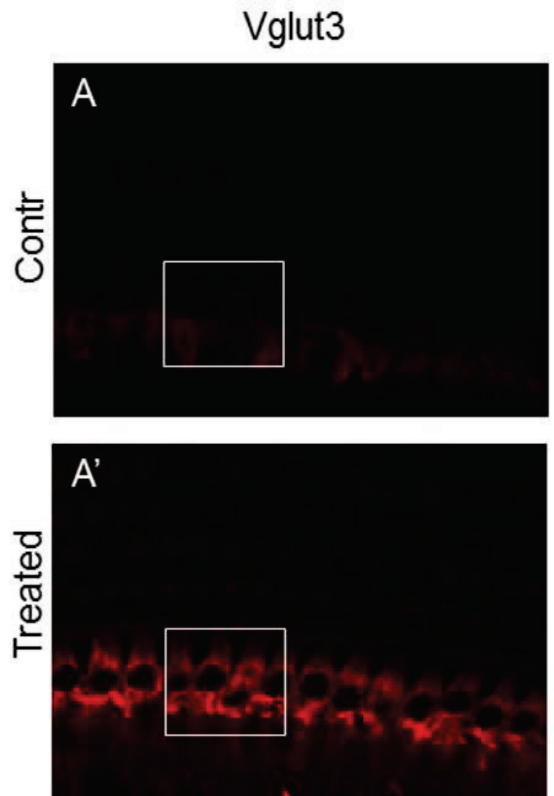

B
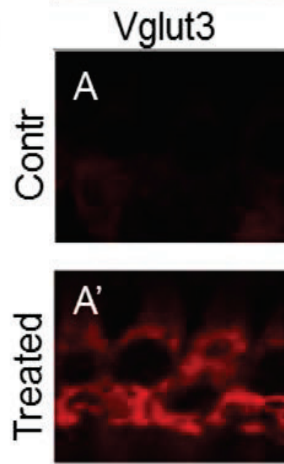

Myosin VIla
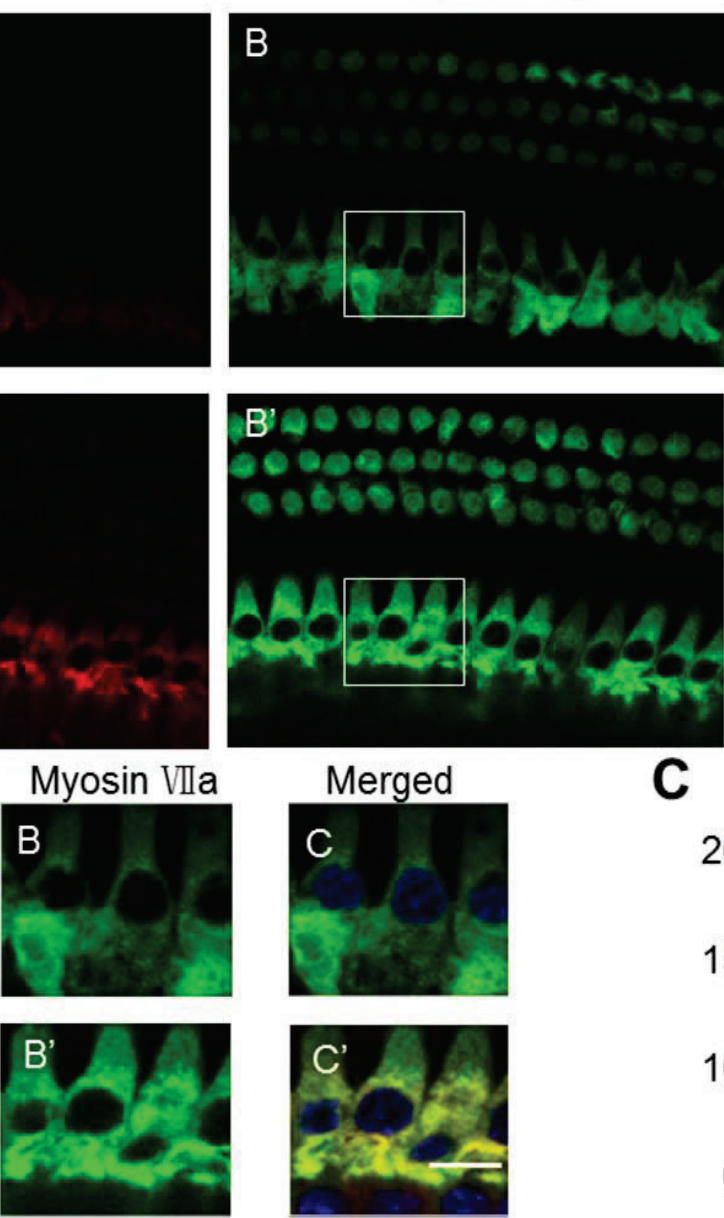
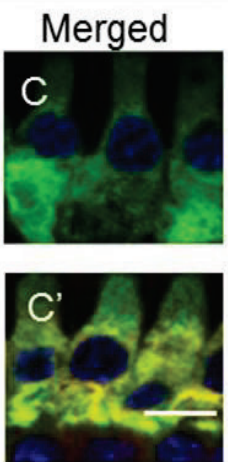

Merged
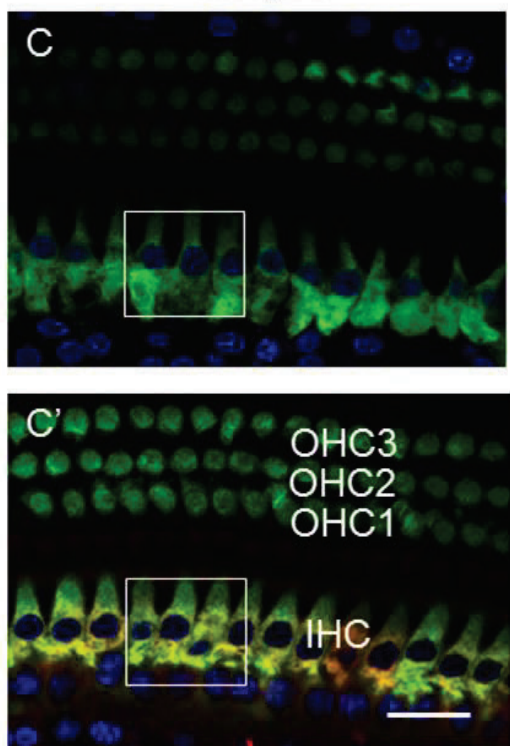

C

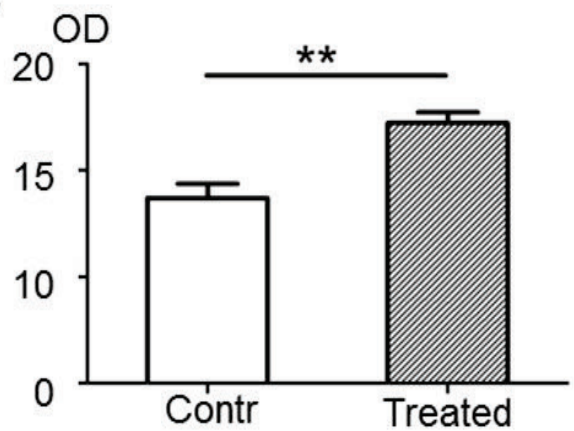

Fig. 3. Immunofluorescence of VGLUT3 expression in the cochlea after salicylate treatment. (A) Overview of whole-mount immunostaining in the control $(n=12)$ and treated groups $(n=12)$ for VGLUT3 (red, A-A'), myosin VIIa (green, B-B'), DAPI (blue), and merged images (C-C') (control group: A-C, treated group: $A^{\prime}-C^{\prime}$ ). Specific VGLUT3 expression can be seen in the IHCs, and there was no detectable signal of VGLUT3 in the OHCs. Bar=20 $\mu \mathrm{m}$. (B) Enlarged images show significant enhancement of VGLUT3 expression in the IHCs from the treated group. Bar $=10 \mu \mathrm{m}$. (C) Fluorescence intensity of staining for VGLUT3 in cochlear IHCs. Optical density (OD) values for VGLUT3 in the IHCs were significantly higher in the treated group than the control group $\left({ }^{* *} p<0.01\right.$; controls, $\mathrm{n}=12$; treated, $\left.\mathrm{n}=12\right)$.

A

Contr

Treated
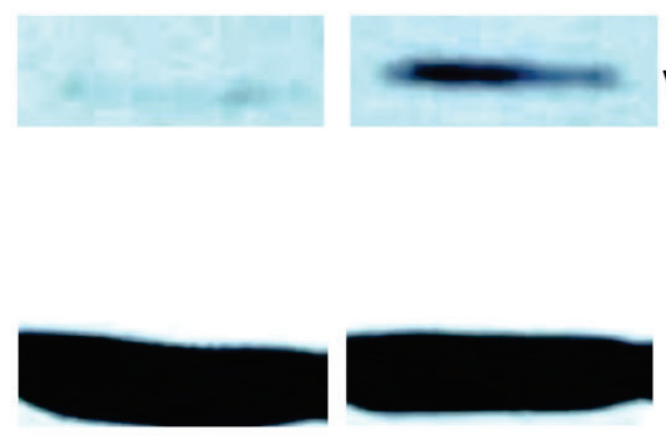

B OD

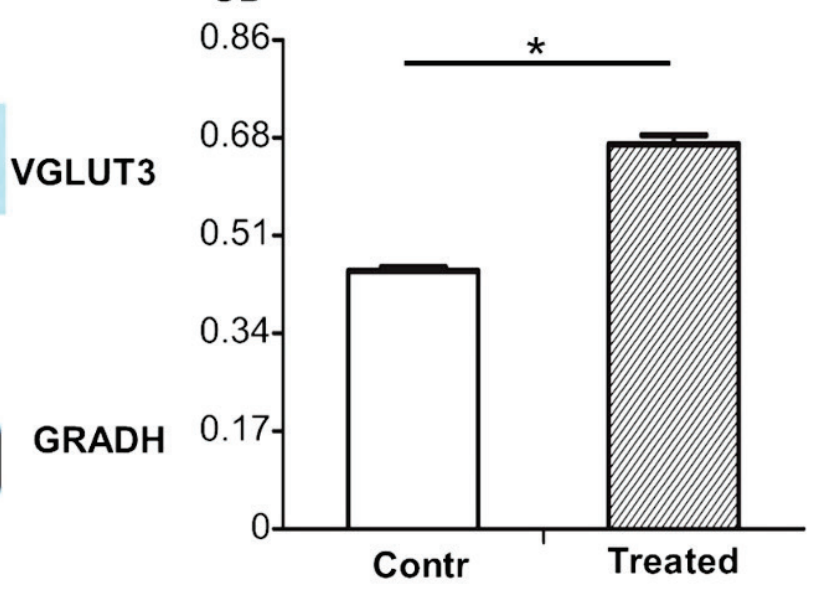

Fig. 4. Quantitative analysis of Western blot showing VGLUT3 expression in the cochlea after salicylate exposure. (A) Western blot showing VGLUT3 expression in the cochlea of the control $(n=12)$ and treated groups $(n=12)$. (B) OD values (VGLUT3/GAPDH) of VGLUT3 expression in the cochlea, as determined by Western blot analysis. Mean OD was significantly higher in the treated rats than the control rats $(p<0.05)$. 
A
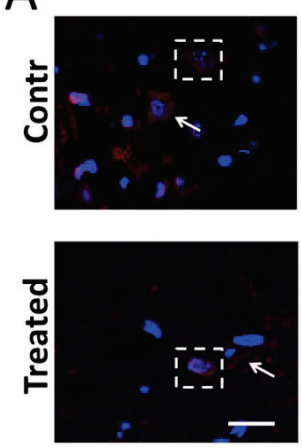

C
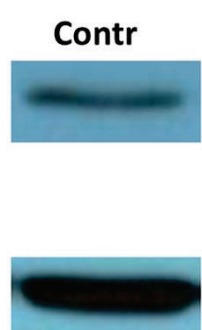
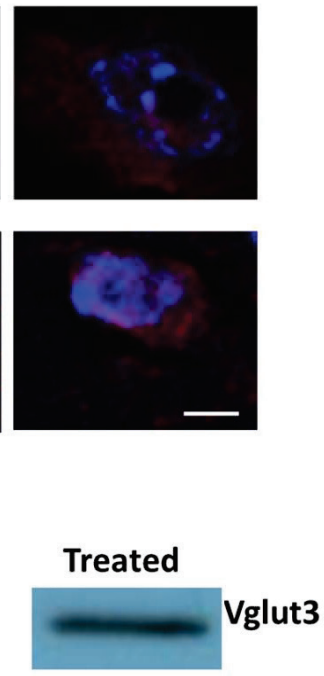

GAPDH
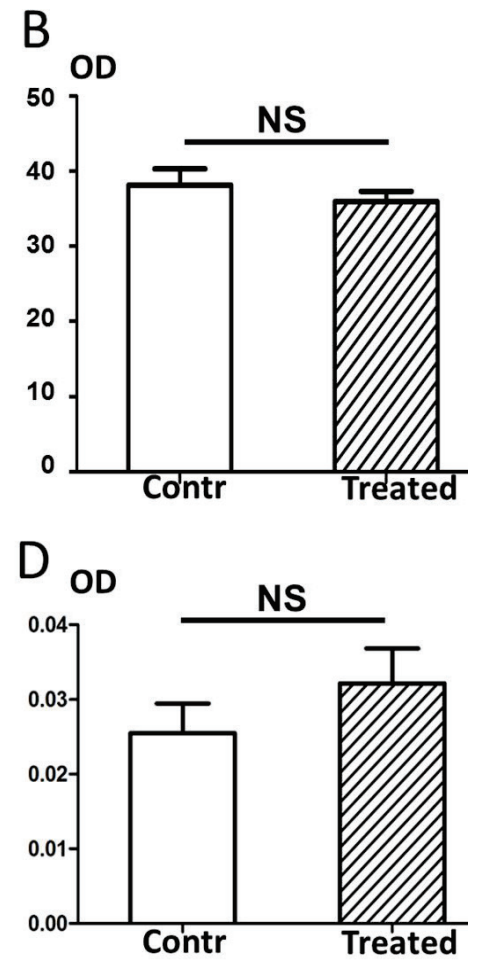

Fig. 5. VGLUT3 expression in the cochlear nucleus after salicylate exposure. (A) VGLUT3 expression in neuronal cells of the cochlear nucleus of rats (red, indicated by the arrow, upper left panel). Cell nuclei were visualized with DAPI staining (blue). Bar $=25 \mu \mathrm{m}$. Enlarged image of the dashed-line frame shows characteristic punctate VGLUT3 staining in the cytoplasm (red, right panel). Bar $=5 \mu \mathrm{m}$. (B) Fluorescence intensity of VGLUT3 in the cochlear nucleus. OD values of VGLUT3 in the cochlear nucleus is similar between the control $(n=6)$ and treated $(n=6)$ groups $(p>0.05)$. (C-D) Western blot of VGLUT3 expression in the cochlear nucleus. OD values (VGLUT3/GAPDH) were similar for the control and treated animals $(p>0.05)$. NS indicates no statistically significant difference.
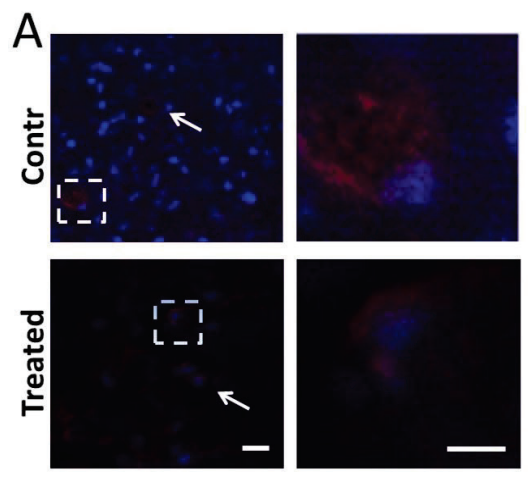

C
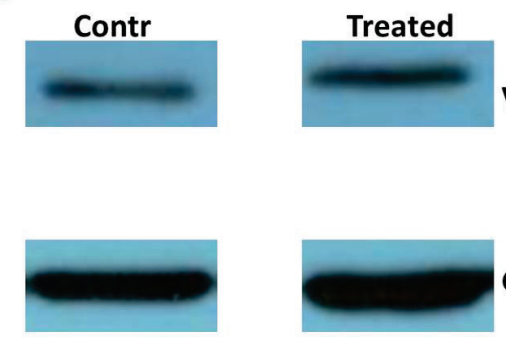

B
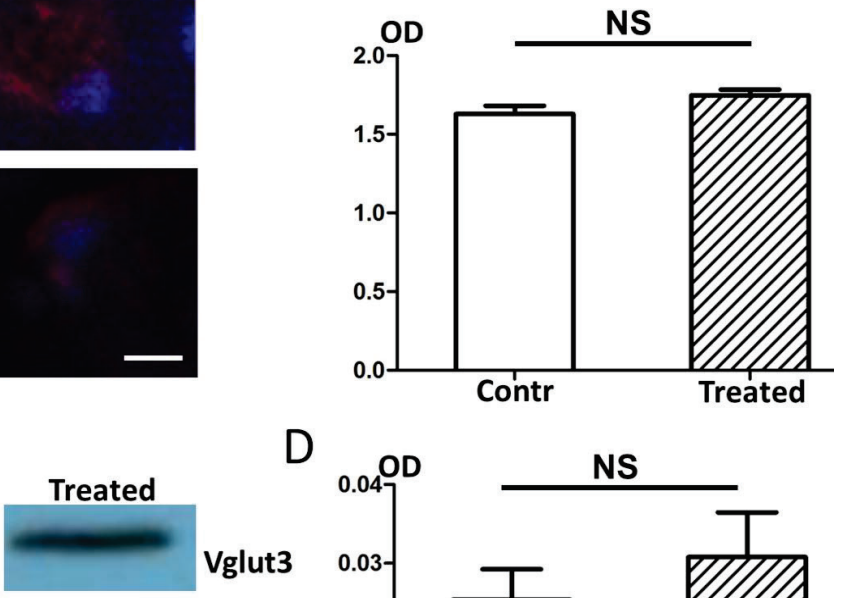

GAPDH

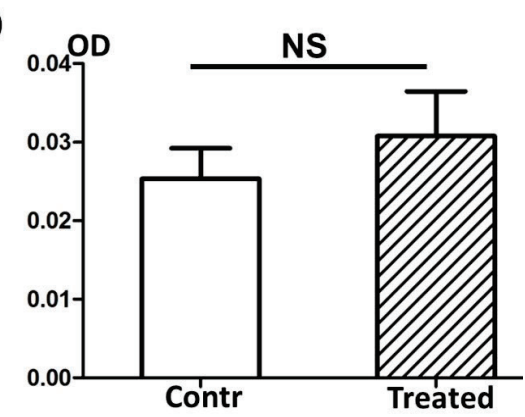

Fig. 6. VGLUT3 expression in the inferior colliculus (IC) after salicylate exposure. (A) VGLUT3 expression in the IC neurons (red, indicated by the arrow, upper left panel). Cell nuclei were visualized with DAPI staining (blue). Bar=10 $\mu$ m. Enlarged image of the dashed line frame shows punctate cytoplasmic VGLUT3 staining (red, upper right panel). Bar $=5 \mu \mathrm{m}$. (B) Fluorescence intensity of VGLUT3 in the ICs. The OD values for VGLUT3 in the IC cells were similar between the control $(n=6)$ and treated $(n=6)$ groups $(p>0.05)$. (C-D) Western blot analysis of VGLUT3 expression in the IC cells yielded similar OD values (VGLUT3/ GAPDH) for the control and treated groups $(p>0.05)$. NS indicates no statistically significant difference. 


\section{Discussion}

The results of the present study indicate that exposure to SS specifically enhances VGLUT3 expression in the cochlear IHCs of rats. The increase in VGLUT3 expression correlated with the generation of tinnitus. However, VGLUT3 expression in the cochlear nucleus and inferior colliculus was unaffected by exposure to SS. This is the first report that VGLUT3 expression in IHCs may contribute to the development of tinnitus. These findings suggest that alteration of VGLUT3 expression in the peripheral auditory system may contribute to the pathogenesis of tinnitus. In this study, treatment with a moderate dose of SS did not significantly elevate the hearing threshold (maximal elevation of ABR threshold was nearly $10 \mathrm{~dB}$ SPL), although significant differences between the treated and control groups were found at some of the frequencies investigated.

VGLUT3, encoded by SLC17a8 and reported to be associated with hearing, was first identified as the third subtype of glutamate vesicular transporter (VGLUT) in 2002 (Takamori 2002). This molecule was proposed to serve as a critical component at the cochlear ribbon synapses. Mice lacking VGLUT3 are congenitally deaf due to the lack of glutamate release at IHC ribbon synapses (Akil et al. 2012). VGLUT3 is responsible for neurotransmitter delivery into synaptic vesicles before it is released into the synaptic cleft. VGLUT3 expression may be altered during development and maturation. Our group previously reported that VGLUT3 expression increased with the development of hearing, and decreased VGLUT3 expression is usually seen in the cochlea of aging rats, indicating a critical role in the auditory function (Peng et al. 2013). Alternatively, the unusual distribution of VGLUT3 suggests a signaling role for glutamate. VGLUT3 may act as a key mediator of glutamate signaling in both peripheral and cortical auditory systems. VGLUT3 is widely expressed in the rat brain and is usually present in the terminals of long projecting neurons (Herzog et al. 2004). VGLUT3 expression in the cortex is localized primarily in GABAergic interneurons and serotonergic projections from raphe nuclei (Seal 2008).

The structure of SS allows it to effectively penetrate the blood-brain barrier and reach high concentrations in the inner ear and brain. However, SS has different effects on the peripheral and central auditory systems (Chen et al. 2013). Salicylate also reduces GABA-mediated inhibition in the lateral amygdala (LA) and other regions of the limbic system (Gong et al. 2008). Previous studies showed that the application of salicylate increased spontaneous activity in the inferior colliculus (Raghavan et al. 2016). High doses of salicylate may induce reversible tinnitus in both patients and animals (Chen et al. 2013) by decreasing cochlear output and enhancing hyper-excitability in regions along the central auditory pathway (Sun et al. 2009). For this reason, SS is used to generate tinnitus in animal models (Baguley et al. 2013, Sun et al. 2015). At high doses, salicylate induces tinnitus that is normally coupled with temporary hearing loss (Chen et al. 2013).

Tinnitus, a phantom sound perception that affects about $10-15 \%$ of the population in the U.S., may be accompanied by hearing loss (Shargorodsky et al. 2010). Tinnitus is often accompanied by reduced sound tolerance, i.e. hyperacusis (Gu et al. 2010). More than $1 \%$ of symptoms require medical treatment or intervention (Baguley et al. 2013). However, the development of effective therapies has been impeded by a lack of understanding of the molecular basis of tinnitus.

One critical concern about the origins of tinnitus is whether the abnormal neural activity initiates in the ear or brain. There have been conflicting reports regarding the possible sources of tinnitus. In general, tinnitus is induced by an imbalance in the release of excitatory vs. inhibitory neurotransmitters. Such an imbalance is likely to be activated by changes in auditory and non-auditory neural networks following initial damage to the cochlea (Chen et al. 2013). Previous reports have indicated that SS-induced tinnitus may result from effects on the central auditory system. For example, SS may cause tinnitus by altering intrinsic neuronal properties and reducing synaptic transmission in the medial geniculate body (MGB), resulting in abnormal thalamic output to the auditory cortex (Yan et al. 2012).

Salicylate-induced tinnitus may be mediated by the marked activation of NMDA receptors in the cochlea. Previously, Guitton et al. (2007) reported that blockade of NMDA channels may prevent the development of tinnitus in rats, suggesting that tinnitus may be generated within the cochlea (Ruel et al. 2008). Other studies showed that salicylate might reduce inhibitory postsynaptic activity, selectively downregulating the firing of GABAergic interneurons (Raghavan et al. 2016). This downregulation results in increased acoustic cortex excitability and contributes to the generation of tinnitus. Salicylate was also found to enhance 
hippocampal excitability, suggesting that the limbic system plays a key role in the development of tinnitus (Gong et al. 2008).

Recent evidence has suggested that a moderate dose of salicylate may induce tinnitus via disruption of regular neural activity in the brain (Sun et al. 2015). However, this assertion is not consistent with the results of our study, suggesting that changes in VGLUT3 expression in cochlear IHCs are likely to contribute to salicylate-induced tinnitus. This discrepancy may reflect an underestimation of the severity of changes in the peripheral auditory system. Similar observations have been reported that salicylate-induced tinnitus could stem from cochlear damage. For example, salicylate was shown to alter membrane conductance, as well as $\mathrm{OHC}$ morphology, in vitro (Shehata et al. 1991). Application of SS was reported to significantly reduce compound action potential (CAP) amplitude (Sun et al. 2015).

The results of the present study suggest that abnormal neural activity in the peripheral auditory system contributes to the generation of tinnitus. Our study showed that a moderate dose of salicylate did not disrupt VGLUT3 expression in the cochlear nucleus or auditory cortex, indicating that VGLUT3 expression in these regions does not contribute to the generation of tinnitus. It should be noted that our observations do not preclude an association between brain expression of VGLUT3 and tinnitus, as we did not measure VGLUT3 levels in other parts of the brain known to be involved in the neural networks that mediate tinnitus, such as the medial geniculate body, lateral amygdala, or amygdala (Yu 2015). Salicylate exposure tends to induce hearing loss coupled with tinnitus, suggesting a possible cochlear origin of tinnitus. It has been suggested that salicylate treatment significantly reduces CAP neural output at suprathreshold intensities, and that salicylate binds competitively to the specific key protein in cochlear $\mathrm{OHCs}$ (prestin), resulting in the disruption of $\mathrm{OHC}$ electromotility and distortion product otoacoustic emissions (DPOAE).

In summary, the present study demonstrates that alterations of VGLUT3 expression in cochlear IHCs contribute to the development of tinnitus. Salicylateinduced tinnitus does not require changes in VGLUT3 expression in the cochlear nucleus or auditory cortex. However, it remains unclear whether VGLUT3 levels in other compartments of the brain are affected by salicylate exposure, and if so, whether changes in VGLUT3 expression contribute to tinnitus. Further studies are needed to address these issues.

\section{Conflict of Interest}

There is no conflict of interest.

\section{Acknowledgements}

This study was supported by the National Natural Science Foundation of China (Grant No. 81500789 and 81700917), Beijing Natural Science Foundation (Grant No. 7174291) and China Postdoctoral Science Foundation (Grant No. 2016M601067).

\section{References}

AKIL O, SEAL RP, BURKE K, WANG C, ALEMI A, DURING M, EDWARDS RH, LUSTIG LR: Restoration of hearing in the VGLUT3 knockout mouse using virally mediated gene therapy. Neuron 75: 283-293, 2012. https://doi.org/10.1016/j.neuron.2012.05.019

AMARJARGAL N, ANDEEVA N, GROSS J, HAUPT H, FUCHS J, SZCZEPEK AJ, MAZUREK B: Differential vulnerability of outer and inner hair cells during and after oxygen-glucose deprivation in organotypic cultures of newborn rats. Physiol Res 58: 895-902, 2009.

BAGULEY D, MCFERRAN D, HALL D: Tinnitus. Lancet 382: 1600-1607, 2013. https://doi.org/10.1016/S0140$\underline{6736(13) 60142-7}$

BAUER CA, BROZOSKI TJ, HOLDER TM, CASPARY DM: Effects of chronic salicylate on GABAergic activity in rat inferior colliculus. Hear Res 147: 175-182, 2000. https://doi.org/10.1016/S0378-5955(00)00130-1

CHEN GD, STOLZBERG D, LOBARINAS E, SUN W, DING D, SALVI R: Salicylate-induced cochlear impairments, cortical hyperactivity and re-tuning, and tinnitus. Hear Res 295: 100-113, 2013. https://doi.org/10.1016/j.heares.2012.11.016

CHEN YC, LI X, LIU L, WANG J, LU CQ, YANG M, JIAO Y, ZANG FC, RADZIWON K, CHEN GD, SUN W, KRISHNAN MUTHAIAH VP, SALVI R, TENG GJ: Tinnitus and hyperacusis involve hyperactivity and enhanced connnectivity in auditory-limbic-arousal-cerebellar network. eLife 4: 17, 2015. https://doi.org/10.7554/eLife.06576 
LOBARINAS E, HAYES SH, ALLMAN BL: The gap startle paradigm for tinnitus screening in animal models limitations and optimization. Hear Res 295: 150-160, 2013. https://doi.org/10.1016/j.heares.2012.06.001

GONG N, ZHANG M, ZHANG XB, CHEN L, SUN GC, XU TL: The aspirin metabolite salicylate enhances neuronal excitation in rat hippocampal CA1 area through reducing GABAergic inhibition. Neuropharmacology 54: 454-463, 2018. https://doi.org/10.1016/j.neuropharm.2007.10.017

GU JW, HALPIN CF, NAM EC, LEVINE RA, MELCHER JR: Tinnitus, diminished sound-level tolerance, and elevated auditory activity in humans with clinically normal hearing sensitivity. J Neurophysiol 104: 3361-3370, 2010. https://doi.org/10.1152/jn.00226.2010

GUITTON MJ, DUDAI Y: Blockade of cochlear NMDA receptors prevents long-term tinnitus during a brief consolidation window after acoustic trauma. Neural Plast 2007: 80904, 2007. https://doi.org/10.1155/2007/80904

HERZOG E, GILCHRIST J, GRAS C, MUZERELLE A, RAVASSARD P, GIROS B, GASPAR P, EL MESTIKAWY S: Localization of VGLUT3, the vesicular glutamate transporter type 3, in the rat brain. Neuroscience 123: 983-1002, 2004. https://doi.org/10.1016/j.neuroscience.2003.10.039

LU Y: Metabotropic glutamate receptors in auditory processing. Neuroscience 274: 429-445, 2014. https://doi.org/10.1016/j.neuroscience.2014.05.057

NORENA AJ: Revisiting the cochlear and central mechanisms of tinnitus and therapeutic approaches. Audiol Neurootol 20 (Suppl 1): 53-59, 2015. https://doi.org/10.1159/000380749

NORENA A, FARLEY BJ: Tinnitus-related neural activity: theories of generation, propagation, and centralization. Hear Res 295: 161-171 2013. https://doi.org/10.1016/j.heares.2012.09.010

PENG Z, WANG GP, ZENG R, GUO JY, CHEN CF, GONG SS: Temporospatial expression and cellular localization of VGLUT3 in the rat cochlea. Brain Res 1537: 100-110, 2013. https://doi.org/10.1016/j.brainres.2013.09.019

RAGHAVAN P, STEVEN A, RATH T, GANDHI D: Advanced neuroimaging of tinnitus. Neuroimaging Clin N Am 26: 301-312, 2016. https://doi.org/10.1016/j.nic.2015.12.008

RICHARDSON BD, BROZOSKI TJ, LING LL, CASPARY DM: Targeting inhibitory neurotransmission in tinnitus. Brain Res 1485: 77-87, 2012. https://doi.org/10.1016/j.brainres.2012.02.014

RUEL J, CHABBERT C, NOUVIAN R, BENDRIS R, EYBALIN M, LEGER CL, BOURIEN J, MERSEL M, PUEL JL: Salicylate enables cochlear arachidonic-acid-sensitive NMDA receptor responses. J Neurosci 28: 7313-7323, 2008. https://doi.org/10.1523/JNEUROSCI.5335-07.2008

SEAL RP, AKIL O, YI E, WEBER CM, GRANT L, YOO J, CLAUSE A, KANDLER K, NOEBELS JL, GLOWATZKI E, LUSTIG LR, EDWARDS RH: Sensorineural deafness and seizures in mice lacking vesicular glutamate transporter 3. Neuron 24: 263-275, 2008. https://doi.org/10.1016/j.neuron.2007.11.032

SHARGORODSKY J, CURHAN GC, FARWELL WR: Prevalence and characteristics of tinnitus among US adults. Am J Med 123: 711-718, 2010. https://doi.org/10.1016/j.amjmed.2010.02.015

SHEHATA WE, BROWNELL WE, DIELER R: Effects of salicylate on shape, electromotility and membrane characteristics of isolated outer hair cells from guinea pig cochlea. Acta Otolaryngol 111: 707-718, 1991. https://doi.org/10.3109/00016489109138403

STENSRUD MJ, CHAUDHRY FA, LEERGAARD TB, BJAALIE JG, GUNDERSEN V: Vesicular glutamate transporter-3 in the rodent brain: vesicular colocalization with vesicular gamma-aminobutyric acid transporter. J Comp Neurol 521: 3042-3056, 2013. https://doi.org/10.1002/cne.23331

SUN W, LIU J, ZHANG C, ZHOU N, MANOHAR S, WINCHESTER W, MIRANDA JA, SALVI RJ: Potassium channel activator attenuates salicylate-induced cochlear hearing loss potentially ameliorating tinnitus. Front Neurol 6: 77, 2015. https://doi.org/10.3389/fneur.2015.00077

SUN W, LU J, STOLZBERG D, GRAY L, DENG A, LOBARINAS E, SALVI RJ: Salicylate increases the gain of the central auditory system. Neuroscience 159: 325-334, 2009. https://doi.org/10.1016/j.neuroscience.2008.12.024

SU YY, LUO B, JIN Y, WU SH, LOBARINAS E, SALVI RJ, CHEN L: Altered neuronal intrinsic properties and reduced synaptic transmission of the rat medial geniculate body in salicylate induced tinnitus. PLoS One 7: e46969, 2012. https://doi.org/10.1371/journal.pone.0046969

TAKAMORI S, MALHERBE P, BROGER C, JAHN R: Molecular cloning and functional characterization of human vesicular glutamate transporter 3. EMBO Rep 3: 798-803, 2002. https://doi.org/10.1093/embo-reports/kvf159 